\title{
Innovative Research on user's experience with xiaokaxiu
}

\author{
Yonghai $\mathrm{Yu}^{1, \mathrm{a}}$, Jiao Chen ${ }^{1, \mathrm{~b}}$ and Jinsong Zhang ${ }^{1, \mathrm{c}}$ \\ ${ }^{1}$ Zhejiang University of Technology 310023 Zhejiang, China; \\ a382249707@qq.com, b2286812305@qq.com, '132197@qq.com
}

Keywords: Xiaokaxiu, user experience, pleasure, innovation.

\begin{abstract}
Xiaokaxiu is a video-shoot application that offers entertainment functionality and helps users record their funny videos. This paper, by analyzing its present situation and users experience, concludes its needs for entertainment and different levels of pleasure, and then puts forward some follow-up innovative ideas for this type of product.
\end{abstract}

\section{Preface}

With the development of mobile internet, mobile apps have extented to all aspects of people's life. Some of them stand out and quickly become popular among users but couldn't get users attention steadily. The same is true with Xiaokaxiu as a phenomenal product despite its soaring popularity in July 7 last year. How to maintain sustainable growth has been the issue for Xiaokaxiu. In this situation, this article attempts to explore emotional design and innovative ideas for this type product from the point of pleasure on users experience.

\section{Present situation}

Xiaokaxiu is a video-shoot application. Users can lip-sync by using dubbing and subtitling provided by the application and share the video on other social platforms.

Soon it was rushing to first place in the list of free apps on App Store in less than two months after online in May last year Its slogan is 'Life plays on stage, You play on performance' and the main target users are 90s whose purpose is to show themselves and want people to recognize them. The focus is 'Show'.

So, what advantages does Xiaokaxiu have in terms of users experience, (1)Xiaokaxiu meets the users desire for performing and making funny things, particularly for those young play full users. They have the desire and love to put themselves into the play for experiencing being a star,and even more they may have chance to act with real stars; besides, the very funny script can release their doubi nature and weirdness mentality. (2)Xiaokaxiu meets users showoff mentality and a sense of achievement by its platform and the sharing mechanism, moreover users will be proud once they get positive comment 'follow or like'. (3)It's very easy and comfortable to use because of its simple design. Users can easily choose dubbing they like, meanwhile Xiaokaxiu provides subtitle to reduce the creation difficulty (4)Social interaction and sense of participation. Xiaokaxiu provides much more room for users creativity, which greatly arouse the users sense of participation. The spreading effects of star's microblog and interacting with friends on Wechat, also formed a social foundation for Xiaokaxiu.

However, Xiaokaxiu also facing the problem of lack of users experience, which will hinder its sustainable development. (1)Xiaokaxiu satisfies the needs for entertainment instead of rigid demand. They like to use it, but they will not use it often. (2) Over repetition lead to weaker novelty and attention. Users can quickly become familiar with this app in their fragmented time also can get bored quickly because of the high repetition rate of its content. (3)Xiaokaxiu cannot form users emotional dependency. Emotion is the third level of Maslow's demand, including personal precipitation and belonging. Personal precipitation refers to users embeding their history and works right here, and making it as their personal space. Group belonging refers to users can get the sense of belonging and 
participation right here. Xiaokaxiu spread its content through social platform, but interactions and content of users are more on the platform, not on Xiaokaxiu.

\section{Analysis on the users experience}

Since the design of users experience should center on users, be loyal to their true feelings. Therefore, research and analysis on the users should be the first step to do the user experience design. So we analysis our target users on the basis of present situation, and mining the inner needs of users from the perspective of the target user.

\subsection{Analysis on the user.}

There are two major target group users:

Playful young guys of 90s who are eager to show and act, advocating parody and dub culture. At the same time, they have very high social activity and social contagion

Stars, they are pretty good at acting and use it for fun. Meanwhile use it to advertise their films and attract followers.

\subsection{Analysis on the user experience.}

We will target the main users (playful young guys) and research user's nee-ds through interviews with ordinary users, we found out that people use it mainly for entertainment and satisfying their desire for acting, easing tensions when working. Xiaokaxiu position itself just as a recreational app. So we analyze user's pleasure by the needs for entertainment. Patrick Jordan is a manager of human factors and designer, and is also the author of the book Designing Pleasurable Products in which he divided pleasure into four catagories physio-pleasure, socio-pleasure, psycho-pleasure, ideo-pleasure

We analyze the pleasure users get from four aspects:

Physio-pleasure. Psycho-pleasure includes the pleasure of sight, hearing, smell, taste and touch. Users getting attracted by funny or beautiful video covers is part of sight pleasure; and the pleasure that the funny dubbing gives is part of hearing pleasure.

Socio-pleasure Social pleasure is gained from interaction with others. Funny videos will generate socio-pleasure when they ' like' by others, or received comment like' you're so good at acting, ought to be a star'. Social interaction and sharing on the hierarchy of behaviors can bring emotional integration and social identity at the reflexive level

Psycho-pleasure. This aspect of pleasure involves user's reaction and mentality when using the product. Newborn Xiaokaxiu videos and jokes give users a sense of novelty. And meet their wacky nature. Simple and easy to use brings users performing pleasure in the process of creation, sharing satisfies users show-off mentality and sense of achievement. Meanwhile, to see stars making funny can also enhance users pleasure. And pleasure and mentality produced in using these products are all experience at behavior level.

Ideo-pleasure. This kind of pleasure is a refection of experience, namely people appreciate the aesthetics and quality of a product, or the product can to some extent improve life and respect the environment Users can demonstrate the ability to perform and establish self image on the platform, can also get satisfaction and social recognition through its social attributes.

\section{Follow-up innovative ideas about this type of product}

How we could sustain the pleasure when using Xiaokaxiu and its follow-up development?

We will still explore the follow-up ideas from four aspects on the basis of Lionel Triger' s pleasure theory. Socio-pleasure, psycho-pleasure, ideo-pleasure belong to behavior levels and reflective levels and deserve more attention than physio-pleasure at visceral level. Moreover, Xiaokaxiu has been doing very well in terms of the physio-pleasure that weirdo covers and the sketch type of voice has successfully attracted users.

Next, we will find ways to sustain the growth from the perspective of socio-pleasure, psycho-pleasure and ideo-pleasure.

Enhancing the socio-pleasure. 
Social needs is an important part in the Pyramid of Maslow's demand. Satisfying the social needs could dramaticlly enhance the user's sense of participation and social pleasure Social needs include the needs of sense of belonging and recognition when interacting with others. So we could enhance the social pleasure by forming the sense of belonging and identity.

(1) Sense of belonging refers to the level of acceptance an individual or group about a matter or phenomenon and the level of intimacy when the matter and phenomenon related Users put their emotions on the product could raise the sense of belonging. Therefore, Xiaokaxiu should shift the third party social platform it relying on to its own platform. Meanwhile, improving the function of inviting friends and search friends, thus forms group belonging of interest circle and network of friends.

(2) Sense of recognition refers to people's useful or valuable judgment and evaluation on themselves and surrounding environment. Human has the need to be recognized in nature, which essentially inspired the user. Users love to continually log into the app and update their own status when they fell being recognized Xiaokaxiu could improve the functions such as bullet screen, thumbs-up, comment and follow to promote the interaction between the user, thereby forming the sense of group belonging.

Enhancing the psycho-pleasure.

Users use products with many emotions and mental activity. Whether or not meeting the needs of users, as well as the practicability of product function, determines the degree of psycho-pleasure the product brings. We should meet the user's demand and strengthen the practicality.

(1) Meeting the demand produces psycho-pleasure. Meeting user's needs and an objective thing that conforms to one' $s$ wishes will produce pleasure. Xiaokaxiu just meet the need for entertainment and should continually add new functions and jokes based on the feedback and needs in using the app, which produce the psycho-pleasure. For example, Xiaokaxiu adds the function co-star according to user's feedback that it's more funny to put on a joint performance.

(2)Practicability can enhance the persistence of psycho-pleasure. Funny and amusing products can only produce short-lived pleasure, but practicability can turn this simple impression into a lasting sense of pleasure. So Xiaokaxiu could extend the psychological pleasure to the point of public life in a way to enhance its practicability. For instance, Xiaokaxiu transfers video into GIF, which is used as chat expressions on Wechat or QQ. In this way, Xiaokaxiu not only enhances the user's pleasure but also raise the adhesiveness of users.

Enhancing ideo-pleasure.

The real level of products is to meet the emotional needs of people on the level of reflection, the most important need is to establish self-image and social status. Xiaokaxiu should help normal users establish the image like 'I'm good at acting, I'm good at making fun. I' $m$ really awesome' and help star users increase their social influence and establish social status, making users proud and thus produce ideo-pleasure.

In view of the above, we propose the method of enhancing socio-pleasure, psycho-pleasure and ideo-pleasure as a follow-up ideas and evaluation criteria.

For Xiaokaxiu, which not only enhance the social attributes but also raise the user's stickiness.

\section{Conclusion}

This article elaborates the entertainment needs of users and analyze pleasure of different levels based on the book Designing Pleasurable Products. and put forward enhancing the socio-pleasure, psycho-pleasure and ideo-pleasure as a way implemented for Xiaokaxiu to sustain user's loveliness.

With the mobile internet develops, app market is lucrative, only those focus on improving users experience and needs could succeed. We focus on the entertainment needs for Xiaokaxiu ,and hopefully this type of product could get sustainable development and enhance the attractiveness of product and user's pleasure from the perspective of the user experience. 


\section{References}

[1]. Philip George Zimbardo, Richard J. Gerrig Wrote Wang lei Translate Psychology and Life Posts and Telecom Press,2003.10.

[2]. Donald Arthur Norman Wrote, Fu qiufang, Chengjinsan Translate Affective Design. Publishing House Of Electronics Industry. 